\title{
A Note on Sums of Four Cubes
}

\author{
By M. Lal, W. Russell and W. J. Blundon
}

\begin{abstract}
A search for the integral solutions of the Diophantine equation $x^{3}+y^{3}+2 z^{3}=k$, for $|x|,|y|$ and $|z|<10^{5}$ was made on an I.B.M. 1620 Model 1 . These results showed that there are now just 19 values of $k$ in the range $1 \leqq k \leqq 999$ for which no solution is known.
\end{abstract}

Sierpiński [4] asks the question, "Can every natural number $k$ be put in the form $x^{3}+y^{3}+2 z^{3}$, where $x, y, z$ are integers?". Partial answers have been given by Ko [1], Makowski [2] as well as by Schinzel and Sierpiński [3]. Ko found a representation for every natural number up to 100 with the exception of the num-

TABLE 1

Integral Solutions of $x^{3}+y^{3}+2 z^{3}=k$.

\begin{tabular}{rrrr}
\hline \multicolumn{1}{c}{$X$} & \multicolumn{1}{c}{$Y$} & \multicolumn{1}{c}{$Z$} & \multicolumn{1}{c}{$K$} \\
1118 & 2723 & -2210 & 99 \\
-2212 & 2549 & -1421 & 99 \\
-2276 & -2627 & 2464 & 229 \\
4636 & -5699 & 3496 & 229 \\
1099 & -1199 & 583 & 274 \\
5185 & -5201 & 865 & 274 \\
1523 & -1525 & 191 & 284 \\
-8413 & 8507 & -2161 & 284 \\
778 & -2072 & 1615 & 454 \\
-4991 & -9710 & 8041 & 571 \\
1168 & -1169 & 127 & 589 \\
3088 & 7168 & -5837 & 598 \\
2926 & 4405 & -3809 & 643 \\
7210 & 7939 & -7592 & 643 \\
-2415 & 2507 & -942 & 692 \\
-2631 & 2653 & -613 & 692 \\
2647 & -3003 & 1622 & 692 \\
3385 & 3955 & -3692 & 724 \\
-1867 & 2318 & -1438 & 725 \\
4931 & 5894 & -5455 & 725 \\
-944 & -1155 & 1060 & 741 \\
-1007 & 8148 & -6463 & 755 \\
680 & 1113 & -946 & 825 \\
-2218 & 2531 & -1384 & 851 \\
2972 & 3371 & -3184 & 851 \\
-1274 & 1849 & -1286 & 913 \\
-8561 & 8626 & -1931 & 913 \\
-9142 & 9587 & -3883 & 941 \\
-4117 & 6521 & -4699 & 950 \\
6220 & 9268 & -8033 & 958 \\
\hline
\end{tabular}

Received July 16, 1968. 
bers 76 and 99 . Makowski investigated the range $101 \leqq k \leqq 220$ and gave representations for all but the cases $k=113,148,183,190,195$.

In the investigations [1], [2] the parameters were small; in fact, the absolute value of each is less than 260 . Since it is possible that some of the missing solutions might be just outside that range, we investigated the problem of finding further solutions of the Diophantine equation

$$
x^{3}+y^{3}+2 z^{3}=k,
$$

where the parameters $|x|,|y|,|z|, k$ were allowed to vary up to 999 .

This search gave representations for all but 39 values of $k$. We note the following three solutions missing in [2].

$$
\begin{aligned}
(-133)^{3}+(-46)^{3}+2 \times(107)^{3} & =113 \\
(-602)^{3}+450^{3}+2 \times(399)^{3} & =190 \\
(-79)^{3}+126^{3}+2 \times(-91)^{3} & =195 .
\end{aligned}
$$

The representation for $k=113$ is reported in [4] to have been found by K. Moszynski and J. Swianiewicz.

On an I.B.M. 1620 Model 1, a Fortran program for this problem took about 15 hours to run. Later on, we rewrote the program in the assembler language which ran about 15 times faster and decided to extend the search to $10^{4}$. This required a computing time of 1000 hours and the run was made over a period of one year on a low priority basis. This extended search gave us solutions for 20 more values of $k$ and they are given in Table 1.

As a result, we now have just 19 values of $k$ in the range $1 \leqq k \leqq 999$ for which no solution of (1) is known. These values are recorded in the Table 2 below.

TABLE 2

Values of $k$ in the Range $1 \leqq k \leqq 999$ for which no Solution of $x^{3}+y^{3}+2 z^{3}=k$ is known.

\begin{tabular}{rlll}
\hline 76 & 356 & 491 & 788 \\
148 & 418 & 519 & 923 \\
183 & 428 & 580 & 931 \\
230 & 445 & 671 & 967 \\
253 & 482 & 734 & \\
\hline
\end{tabular}

The detailed results of our investigations are being prepared for depositing in the Unpublished Mathematical Tables file of this journal.

Department of Mathematics

Memorial University of Newfoundland

St. John's, Newfoundland, Canada.

1. C. Ko, "Decompositions into four cubes," J. London Math. Soc., v. 11, 1936, pp. 218-219.

2. A. Makowski, "Sur quelques problèmes concernant les sommes de quatre cubes," Acta Arith., v. 5, 1959, pp. 121-123. MR 21 \#5609.

3. A. Schinzel \& W. Sierpiński, "Sur les sommes de quatre cubes," Acta Arith., v. 4, 1958, pp. $20-30$.

4. W. Srerpiński, A Selection of Problems in the Theory of Numbers, Macmillan, New York, 1964, p. 115. MR $30 \# 1078$. 